\title{
Detecting companions to extrasolar planets using mutual events
}

\author{
J. Cabrera and J. Schneider
}

\author{
Observatoire de Paris-Meudon, 92195 Meudon Cedex, France \\ e-mail: juan.cabrera@obspm.fr
}

Received 26 July 2006 / Accepted 22 December 2006

\section{ABSTRACT}

\begin{abstract}
Aims. We investigate a new approach to the detection of companions to extrasolar planets beyond the transit method. We discuss the possibility of the existence of "binary planets".

Methods. We develop a method based on the imaging of a planet-companion as an unresolved system (but resolved from its parent star). It makes use of planet-companion "mutual phenomena", namely mutual transits and mutual shadows.

Results. We show that companions can be detected and their radius measured down to lunar sizes.
\end{abstract}

Key words. stars: planetary systems - occultations

\section{Introduction}

The search for satellites of extrasolar planets is relevant to the understanding of the evolution of planetary systems and to the perspective of their habitability. Their occurrence in other planetary systems is very likely since in the Solar System, 7 of the 9 "planets" have from 1 to several tens of satellites and numerical simulations of planet formation show that such satellites should be common (Ida et al. 1997). Several methods have been proposed for their detection: Sartoretti \& Schneider (1999) proposed detection by transits; Brown et al. (2001) applied this approach to constrain companions to HD 209458 b; Han \& Han (2002) and Bennett \& Rhie (2002) discussed the possibility of detecting companions via microlensing; Williams \& Knacke (2004) have shown that Earth-like satellites of giant planets in the habitable zone would be detectable by spectroscopy in the $\mathrm{CH}_{4}$ hole of the planet spectrum in the 1.5-4 $\mu \mathrm{m}$ band. Here we investigate new ways to detect planet satellites based on planet wobble and photometry in direct imaging. First, we discuss the possibility of binary planets and their impact on planet characterization.

In addition to satellites defined as companions with masses and sizes significantly smaller than their parent planets, we suggest that there may exist binary planets with comparable masses and sizes. Such binary planets do not exist in the Solar System. However, the detection of the first 180 other planetary systems has shown much diversity and differences with the Solar System in their characteristics and provided surprises such as very small and highly eccentric orbits. These unexpected findings open the possibility of new configurations such as binary planets. In addition, binarity is not an exception in objects conceptually associated with planets, such as asteroids (Pravec et al. 2006), trans-Neptunian objects (Kern \& Elliot 2006) or brown dwarfs (Stassun et al. 2006). In the same way, refined simulations show the formation of vortices close to planets (Kley 2003) which could in turn form a massive companion to the planet.

An interesting counterargument has recently been put forward (Canup \& Ward 2006) predicting that the mass ratio of the companion to its host planet cannot be larger than about $7 \times 10^{-4}$. It will be interesting to see in the coming decade if this prediction is confirmed by observations. We note that this prediction does not hold for non giant Solar System bodies like the Earth and Pluto.

The presence of companions to planets would have a significant impact on their characterization.

- Planet mass. For binary planets the radial velocity method of detection (RV) gives only the sum $M=M_{1}+M_{2}$ of their (minimum) masses, leading to a false assignment of the mass of individual objects. In particular if a companion detected by RV has a (minimum) mass larger than the standard planet mass upper limit ( 13 Jup. mass), it would be inappropriately discarded as being a brown dwarf and not a planet. The same consideration holds for the astrometric detection of planets.

- Radius and albedo. The future detection of planets by direct imaging will give, in the case of a planet-companion system, only the $\operatorname{sum} F=F_{1}+F_{2}$ of their fluxes:

$\left(F_{\text {Refl }}\right)_{1,2}=A_{1,2} \times R_{1,2}^{2}$ (modulated by an orbital phase) for the reflected flux; and

$\left(F_{\mathrm{Th}}\right)_{1,2}=R_{1,2}^{2} \times T_{1,2}^{4}$ (constant along the orbit) for the thermal emission.

The albedo $A$ of a cold planet cannot be larger than 1; its radius cannot be larger than $\sim 1.1 R_{\text {Jup }}$ (Guillot 2005). Therefore the normalized flux of a planet cannot be larger than the maximum normalized flux:

$F_{\text {max }}=1.2 R_{\text {Jup }}^{2}$ and $F_{\text {max }}=T^{4} 1.2 R_{\text {Jup }}^{2}$

for the reflected and thermal flux respectively.

If the observed normalized flux of the planet is larger than the maximum possible flux $F_{\max }$, either there is something wrong with its structure or it is binary. An example of an odd structure is given by a planet surrounded by rings, since then there is an additional contribution to the planet flux coming from the planetary ring (Schneider 2003). Their presence can be detected in reflected light by a non-Keplerian modulation of their reflected flux along the orbit (Arnold \& Schneider 2004; Barnes \& Fortney 2004); in the thermal regime there is no such orbital modulation and there is a priori no way to disentangle a binary planet from a ringed planet. 
If the observed planet normalized flux is smaller than $F_{\max }$ and if it is in reality a planet-companion system, the assumption that it is a single planet would lead to incorrect assignments to the albedo and/or radius of its components.

- Planet spectrum. If there is a satellite with a significant contribution to the planet flux, the global planet + satellite spectrum may lead to misinterpretations if the spectrum is attributed to a single object (leading to incorrect atmosphere models). An important example is provided by an icy satellite of a telluric planet close to the habitable zone. The planet may undergo a greenhouse effect leading to an atmosphere with water and $\mathrm{CO} 2$, while the satellite can be icy. In that case, photodissociation of the satellite's ice can lead to ozone synthesis (Teolis et al. 2006); the global spectrum would mimic an Earth spectrum.

The case of planets detected by transits is different. Their possible binarity would be easily inferred from the peculiar shape of the transit lightcurve or from their timing revealing non periodic transits (Sartoretti \& Schneider 1999). For the 9 planets detected by transit by 22 Aug. 2005 (HD 209458 b, HD 149026 b, TRES-1, OGLE-TR-10, OGLE-TR-111, OGLETR-113, OGLE-TR-132, OGLE-TR-56, HD 189733; see exoplanet.eu), none of them presents any sign of a moon or of binarity. But their orbital radius is so small $(a<0.05 \mathrm{AU})$ that the Hill radius $a\left(M_{\mathrm{pl}} / 3 M_{*}\right)^{1 / 3}$ (Valtonen 2006) inside which a stable orbit can survive around the planet would make these planets merge together (for a study of the stability of satellites around giant extrasolar planets, see Barnes \& O'Brien 2002). Binarity among "hot Jupiters" is therefore very unlikely. Only planets at orbital distances larger than $\sim 0.1$ AU can, from a dynamical stability point of view, be binary. But the probability that they make a transit drops as $1 / a$ and only large surveys like the CoRoT and Kepler space missions will detect them. Finally, the detection by transits of a binary planet lacks generality since it requires a transit to occur.

Thus, the detection of planets by RV, astrometry and direct imaging miss their possible binarity and could lead to misassignments of their mass, albedo and radius. The most radical method to detected the binarity of a planet would consist of resolving the planet-companion system in high angular resolution direct imaging. But, for a typical Jupiter/Ganymede system at $5 \mathrm{pc}$, the baseline $B$ required to resolve the system at $2 \mu \mathrm{m}$ would be $B=1.2 \times 2 \mu \mathrm{m} \times 5 \mathrm{pc} / 10^{6} \mathrm{~km}=360 \mathrm{~m}$. In addition, a very high contrast of at least $10^{6}$ is required. Such a baseline and high contrast only will be achieved in the future, here we investigate less difficult methods.

\section{New approaches to planet-companion system detection}

We consider the detection of a planet-companion system by direct imaging in which the system is unresolved and thus appears as a single point, supposed to be detached from its parent star. Gaidos et al. (2006) have considered the thermal emission approach. Here we concentrate on the reflected light approach of planet imaging.

\subsection{Planet wobble}

Let $F_{\mathrm{pl}}=\pi A_{\mathrm{pl}} R_{\mathrm{pl}}^{2}$ and $F_{\mathrm{c}}=\pi A_{\mathrm{c}} R_{\mathrm{c}}^{2}$ be the flux of the two components of the planet-companion system. The photocenter will then make a wobble around the center of mass with an amplitude $\Delta a$ given by

$\Delta a=\frac{a_{\mathrm{c}}}{M_{\mathrm{pl}}+M_{\mathrm{c}}}\left(\frac{F_{\mathrm{pl}} M_{\mathrm{c}}-F_{\mathrm{c}} M_{\mathrm{pl}}}{F_{\mathrm{pl}}+F_{\mathrm{c}}}\right)$.

For a Saturn-Titan system, the mass and flux ratios are $M_{\mathrm{c}} / M_{\mathrm{pl}}=$ 0.01 and $F_{\mathrm{c}} / F_{\mathrm{pl}}=0.001$; then at $5 \mathrm{pc}$, the amplitude of the angular wobble is 15 microarcsec. For a Jupiter-Saturn binary system with the same separation $M_{\mathrm{c}} / M_{\mathrm{pl}}=0.3$ and $F_{\mathrm{c}} / F_{\mathrm{pl}}=1$; the angular wobble amplitude is then 1 mas. For a perfect twin planet (same mass, radius and albedo), the photocenter remains fixed along its revolution and the binarity is undetectable by this approach.

These numbers should be compared to the accuracy $\delta \theta$ on the planet position from ground and space observations. This accuracy is given, at best, at a wavelength $\lambda$, for a bandwidth $W$ and a telescope of diameter $d$ by:

$\delta \theta=\frac{1}{\sqrt{N_{\mathrm{ph}}}} \frac{\lambda}{d}$

where $N_{\mathrm{ph}}=10^{3} \times 10^{-0.4 m}(d / 1 \mathrm{~cm})^{2}(W / 1 \AA)\left(T_{\exp } / 1 \mathrm{~s}\right)$ is the number of detected photons emitted by the planet. For a $m=25$ planet, $\delta \theta=15 \mu$ as (resp. $1 \mu$ as) in a $10 \mathrm{~h}$ exposure with $W=100 \mathrm{~nm}$ and $d=8 \mathrm{~m}$ (resp. $d=30 \mathrm{~m}$ ) at $\lambda=1 \mu \mathrm{m}$.

Of course, any intrinsic flux variation of the planet and/or companion would complicate the detection of the signal.

The planet-companion system can be seen as a spectroscopic binary. Each component has a radial velocity variation with an amplitude $V_{1,2}=\left[M_{2,1} /\left(M_{1}+M_{2}\right)\right]\left(G\left(M_{1}+M_{2}\right) / a_{\mathrm{c}}\right)^{1 / 2}$.

For a Saturn-Titan system, the exo-Saturn velocity is $2 \mathrm{~m} / \mathrm{s}$. For a Jupiter-Saturn binary system with the same separation the exo-Jupiter velocity is $10 \mathrm{~km} \mathrm{~s}^{-1}$.

The feasibility of this wobble approach has been addressed in the context of Extremely Large Telescopes by Ardeberg (2005).

\subsection{Mutual photometric phenomena}

Let us consider a restricted three body problem consisting of a star, a planet orbiting the star and a companion orbiting the planet. In our problem, the companion (with radius $R_{\mathrm{c}}$ and mass $M_{\mathrm{c}}$ ) and the planet (with radius $R_{\mathrm{pl}}$ and mass $M_{\mathrm{pl}}$ ) orbit each other in a circular orbit of semimajor axis $a_{\mathrm{c}}$; the center of mass of this system orbits the star (with radius $R_{*}$ and mass $M_{*}$ ) with a semimajor axis of $a_{\mathrm{pl}}$ (see Fig. 1).

The only source of light is the star, which shines with constant luminosity $P$ (following the notation of Lester et al. 1979). The flux (density) $F_{*}$ arriving at the planet and the companion is constant and uniform. The flux reflected by these bodies is not resolved, but it is detached from the light coming from the star. This reflected flux depends on the physical characteristics of the reflecting surfaces and the geometrical configuration of the system.

The theory that describes the radiation by a planet illuminated by its star is well described in Lester et al. (1979) and Fairbairn (2002) for example. Here we will suppose that both the planet and the satellite are Lambertian spheres. Scattering atmospheres may differ from this model but ours is still a good approximation. 


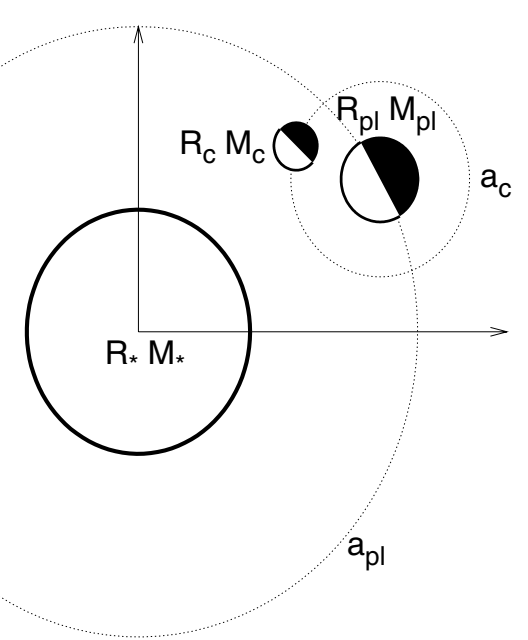

Fig. 1. Schematic view of the system (not to scale). The companion's orbit around the planet and planet's orbit around the star do not need to be coplanar. The white surfaces of the planet and the companion are illuminated, while black surfaces are in darkness.

In these circumstances, the flux reflected by the planet (see Eq. (4)) is proportional to:

- its geometrical albedo $p$;

- its surface (that is, the square of the radius $R$ );

- the inverse of the square of its distance to the star $a$;

- the phase law $\Psi(\alpha)$.

The phase law is an integral which takes into account the geometry of the system. It depends on the phase angle $\alpha$, which is the angle between the incident direction of the radiation and the direction to the observer, as seen from the center of the sphere (see Fig. 2). The integral has the following form:

$\Psi(\alpha)=\frac{3}{2 \pi} \iint \mathrm{d} \theta \mathrm{d} \phi \sin ^{3}(\theta) \cos (\phi) \cos (\phi+\alpha)$.

With these assumptions, the flux reflected by the planet as seen from Earth is:

$F_{\mathrm{pl}}=\mathrm{p} \frac{R_{\mathrm{pl}}^{2}}{a_{\mathrm{pl}}^{2}} \Psi(\alpha) F_{*}$.

The limits of integration of Eq. (3) are $\theta \in[0, \pi]$ and $\phi \in$ $[-\pi / 2, \pi / 2+\alpha]$; so it takes the form:

$\Psi(\alpha)=\frac{\sin (\alpha)+(\pi-\alpha) \cos (\alpha)}{\pi}$.

For further details on how to arrive at these expressions, please see Lester et al. (1979).

In general, the total flux that we measure is the addition of the individual fluxes coming from each body. However, if the orbit of the satellite lies on the line of sight of the observer, the orbital revolution of the satellite around the planet has 4 remarkable phases (see Fig. 3):

- Phase 1: companion's shadow on the planet;

- Phase 2: companion transiting in front of the planet;

- Phase 3: companion eclipsed by the planet;

- Phase 4: companion passing behind the planet and being occulted by it.

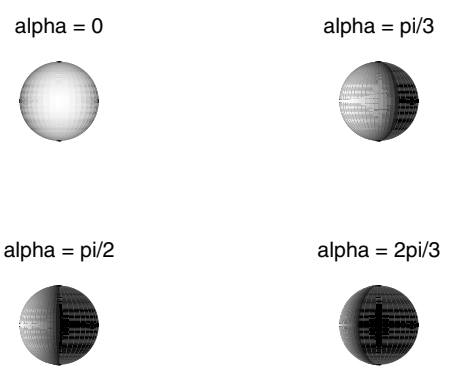

Fig. 2. Illumination of the planet as seen from the observer for different phase angles.
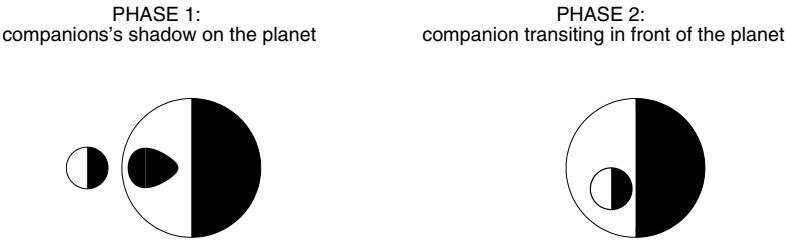

PHASE 3:
companion eclipsed by the planet

PHASE 4:

companion occulted by the planet
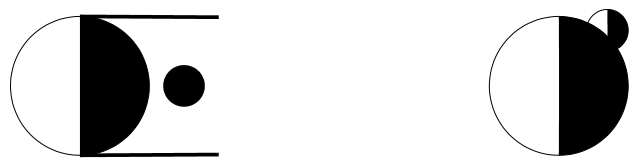

Fig. 3. Mutual phenomena during the revolution of a companion around its parent planet; seen from the point of view of the observer; illumination comes from the left.

In any of these cases we see a flux decrease because part of the reflecting surface is in darkness.

To calculate this flux decrease we consider the surface in darkness and we integrate Eq. (4) within the limits of this surface. We have calculated this flux numerically and we present it in Figs. 4 to 7 for different configurations of the system. In the next subsections we will detail these calculations. The signal in the lightcurves is not periodic any more (as it was in the transits of a single planet in front of a star); the position, shape and depth of the flux decrease strongly depend on the relative positions of all the bodies involved (star, planet, companion and observer).

\section{Mutual transits}

One of the bodies is transiting in front of the other, occulting its surface. This surface is the intersection of a cylinder with a sphere: a classic problem of surface geometry. However, the expression resulting from integration (4) within the limits imposed by the intersection is not analytic. In the next two points we will approximate that integral for the two possible transits, in order of difficulty.

The geometric probability of transits is $R_{\mathrm{pl}} / a_{\mathrm{c}} \approx 10 \%$.

\section{- Phase 4: companion occulted by the planet}

The companion is supposed to be smaller than the planet, so except in the ingress and egress phases, the companion is 

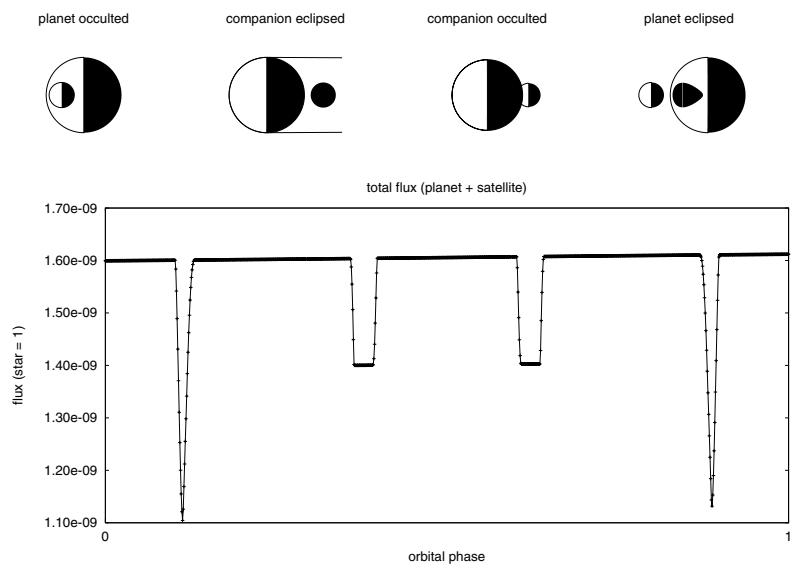

Fig. 4. Lightcurve for a planet with a companion; the phase angle is $\alpha=90^{\circ} ; R_{\mathrm{c}} / R_{\mathrm{pl}}=1 / 3$; the trend in the out-of-event part of the curve is due to the variation in phase of the planet, as it orbits the star.

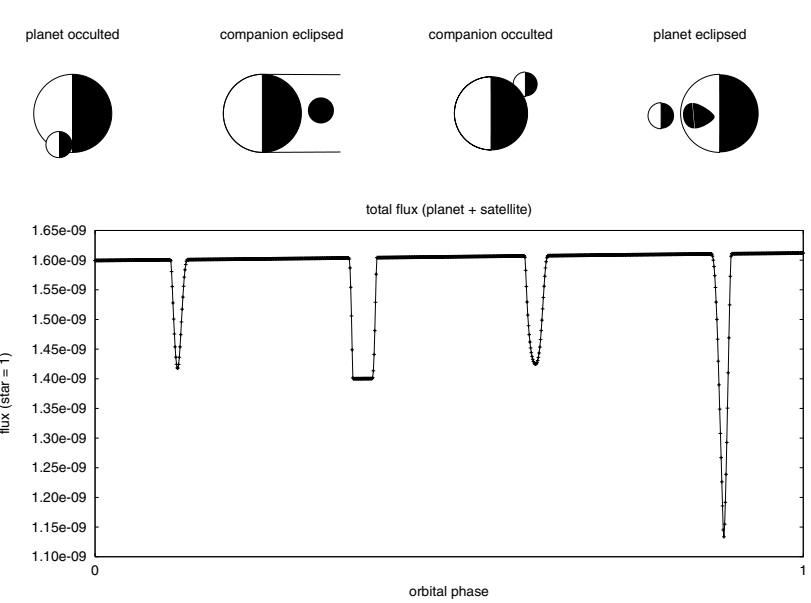

Fig. 5. Lightcurve for a planet with a companion; the phase angle is $\alpha=90^{\circ} ; R_{\mathrm{c}} / R_{\mathrm{pl}}=1 / 3$; the orbit of the companion is inclined $85^{\circ}$ with respect to the line of sight.

completely occulted ${ }^{1}$. Using Eq. (4), the corresponding flux decrease is:

$\frac{\Delta F_{\mathrm{T}}}{F_{\mathrm{T}}}=\frac{F_{\mathrm{c}}}{F_{\mathrm{pl}}+F_{\mathrm{c}}}=\frac{p_{\mathrm{c}} R_{\mathrm{c}}^{2}}{p_{\mathrm{pl}} R_{\mathrm{pl}}^{2}+p_{\mathrm{c}} R_{\mathrm{c}}^{2}}$.

The duration of this transit is:

$\Delta T_{4}=\frac{P_{\mathrm{c}} R_{\mathrm{pl}}}{\pi a_{\mathrm{c}}}$

\section{- Phase 2: companion transiting in front of the planet}

We can consider the maximal occultation: this is, the maximum (or minimum, depending on the reader's choice) of the flux decrease. To do so, we suppose that the surface occulted ${ }^{2}$ has a constant brightness (which is not true) and we approximate this

\footnotetext{
${ }^{1}$ If the plane of the orbit of the satellite is inclined, this may not be true (see Fig. 5); in that case we will have to calculate which is exactly the occulted surface of the companion.

${ }^{2}$ Regarding Eq. (3) we can easily verify that the coordinates of the brightest point of the surface are $\theta=\pi / 2$ and $\phi=-\alpha / 2$.
}

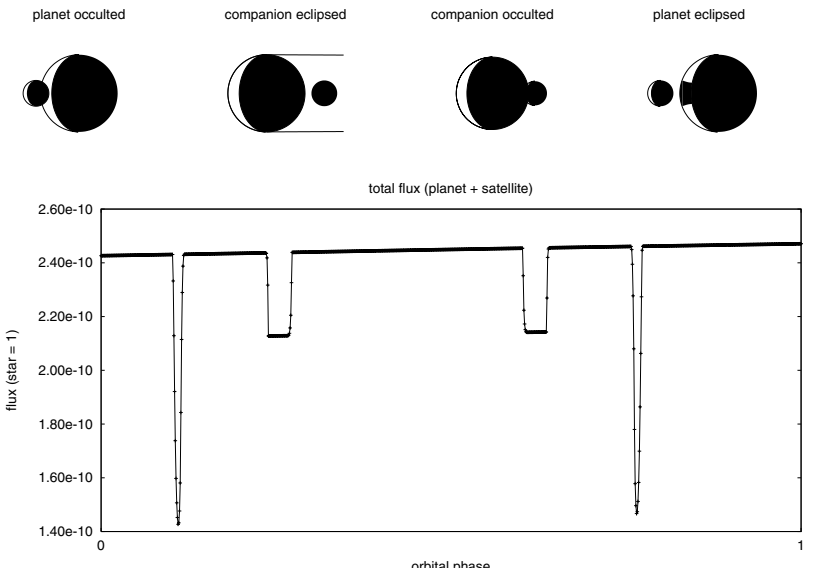

Fig. 6. Lightcurve for a planet with a companion; the phase angle is $\alpha=135^{\circ} ; R_{\mathrm{c}} / R_{\mathrm{pl}}=1 / 3$.

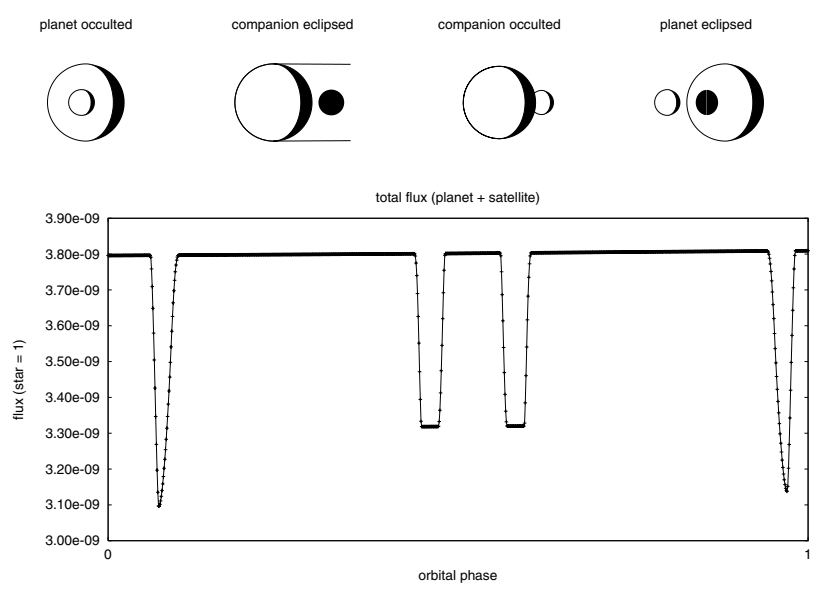

Fig. 7. Lightcurves for a planet with a companion; the phase angle is $\alpha=45^{\circ} ; R_{\mathrm{c}} / R_{\mathrm{pl}}=1 / 3$.

area by the surface of a disk with the companion's radius. The corresponding flux decrease is:

$$
\frac{\Delta F_{\mathrm{T}}}{F_{\mathrm{T}}} \approx \frac{p_{\mathrm{pl}} R_{\mathrm{c}}^{2} \frac{3}{2} \cos \alpha / 2}{\left(p_{\mathrm{pl}} R_{\mathrm{pl}}^{2}+p_{\mathrm{c}} R_{\mathrm{c}}^{2}\right) \Psi(\alpha)}
$$

and the event duration is:

$$
\Delta T_{2}=\frac{P_{\mathrm{c}} R_{\mathrm{pl}}(1+\cos \alpha)}{2 \pi a_{\mathrm{c}}} .
$$

\section{Mutual shadows}

In phases 1 and 3 one body eclipses the other. In eclipses we have to differentiate the umbra (defined as the region of an eclipse where the light coming from the primary source is completely excluded) from the penumbra (the region where only part of the light from the primary source is excluded). However, in the case of planets orbiting at distances similar to those of Jupiter or Saturn, the fraction of time in which the companion is only in penumbra compared with the fraction of time in which it is in umbra is of the order of $10^{-4}$ (whereas for the Earth-Moon system it is 0.7); so we decided to neglect $\mathrm{it}^{3}$.

\footnotetext{
${ }^{3}$ The effect of penumbra will be to soften the sharpness of the egress and ingress, which are very difficult to observe anyway.
} 
In this case the surface of interest is the intersection of a cone with a sphere. In the next two points we will approximate the integral (3) for the eclipses.

If the companion's orbital plane is close to the planet ecliptic, the geometric probability of mutual shadows is close to $100 \%$.

\section{- Phase 3: companion eclipsed by the planet}

The companion disappears completely in the shadow of the parent planet. The relative flux variation is then:

$\frac{\Delta F_{\mathrm{T}}}{F_{\mathrm{T}}}=\frac{F_{\mathrm{c}}}{F_{\mathrm{pl}}+F_{\mathrm{c}}}=\frac{p_{\mathrm{c}} R_{\mathrm{c}}^{2}}{p_{\mathrm{pl}} R_{\mathrm{pl}}^{2}+p_{\mathrm{c}} R_{\mathrm{c}}^{2}}$

with a duration of:

$\Delta T_{3}=\frac{P_{\mathrm{c}} R_{\mathrm{pl}}^{\prime}}{\pi a_{\mathrm{c}}}$

$R_{\mathrm{pl}}^{\prime}$ is the radius of the perpendicular section of the planet's shadow cone at the position of the companion:

$R_{\mathrm{pl}}^{\prime}=R_{\mathrm{pl}}-\frac{R_{*}-R_{\mathrm{pl}}}{a_{\mathrm{pl}}} a_{\mathrm{c}}=R_{\mathrm{pl}}\left(1-\frac{R_{*}-R_{\mathrm{pl}}}{R_{\mathrm{pl}}} \frac{a_{\mathrm{c}}}{a_{\mathrm{pl}}}\right)$.

\section{- Phase 1: satellite's shadow on the planet}

In this case the companion projects a shadow on the surface of the planet. To calculate the flux decrease we proceed as in phase 2 and we obtain:

$$
\frac{\Delta F_{\mathrm{T}}}{F_{\mathrm{T}}} \approx \frac{p_{\mathrm{pl}} R_{\mathrm{c}}^{\prime 2} \frac{3}{2} \cos \alpha / 2}{\left(p_{\mathrm{pl}} R_{\mathrm{pl}}^{2}+p_{\mathrm{c}} R_{\mathrm{c}}^{2}\right) \Psi(\alpha)}
$$

where $R_{\mathrm{c}}^{\prime}$ is calculated in the same way as $R_{\mathrm{pl}}^{\prime}$ in phase 3 . The duration of the passage is:

$\Delta T_{1}=\frac{P_{\mathrm{c}} R_{\mathrm{pl}}(1+\cos \alpha)}{2 \pi a_{\mathrm{c}}}$.

The light curves presented here are free of noise, which obviously would make the detection of these events more difficult.

\section{Mutual phenomena in the thermal regime}

In the thermal regime, where one detects the thermal emission of a planet and its companion, mutual transits occur in the same way as for reflected light and the corresponding light curves have the same shapes. For shadows and eclipses the situation is different. The flux decrease is related to the decrease in temperature of the part of the planet and its companion not illuminated by the parent star. This drop in temperature is sudden when the planet or companion surface is solid (such as during the lunar eclipses). The flux decrease is then identical to the reflected light case. When the planet or its companion have a thick atmosphere, its thermal inertia and atmospheric circulation inhibit the temperature drop and no flux decrease is seen. For thin atmospheres the situation is intermediate. The amount of thermal infrared flux decrease during shadows and eclipses thus provides a way to estimate the thermal inertia of the surface of the planet and its companion (Spencer 1987).

\section{Feasibility and search strategy}

\subsection{Photometric accuracy and detection limits}

For a companion to be detectable, the photometric accuracy must be better than the transit or shadow decrease in an exposure time shorter than, say, half the duration of the phenomenon. The durations are typically of the order of $P_{\text {comp }} R_{\mathrm{pl}} / \pi a_{\text {comp }}$, i.e. $8 \mathrm{~h}$ for a Saturn-Titan system and $4 \mathrm{~h}$ for an Earth-Moon system. The photometric accuracy on the detection of a planet is not controlled by its own photon noise, but by the photon noise of the background consisting essentially, in real situations, of the speckles of the parent star halo.

In current coronagraphic detection projects of planets in the visible, the speckle background is typically 100 times the planet signal, at an angular separation of 1.5 to $2 \lambda / D(D=$ telescope diameter) for a $m=5$ star and coronagraphic rejection factor of the star of 50000 on the stellar peak. This figure holds for Jupiterlike planets at $1 \mathrm{AU}$ (planet to star flux ration $10^{-8}$ ) detected by a $1.5 \mathrm{~m}$ telescope and for Earth-like planets at $1 \mathrm{AU}$ (planet to star flux ratio $10^{-10}$ ) detected by a $7.5 \mathrm{~m}$ TPF-C.

For a companion with one third the planet radius the relative flux decrease during shadows and transits is $\sim 10 \%$, i.e. $10^{-3}$ the speckle background. The detector must collect $10^{7}$ speckle + planet photons to detect the companion with a $S N R=3$ in the speckle background. For a Jupiter-sized planet the accumulated exposure time required is $30 \mathrm{~h}$ for a $m=5 \mathrm{star}$ with a $1.5 \mathrm{~m}$ telescope. Since this exposure time exceeds the duration of the event ( $8 \mathrm{~h}$ in case of a Saturn/Titan-like system) by a factor of 4 , the companion is detectable in a continuous planet monitoring only in cumulative exposures over 4 revolutions around the planet, i.e. after 2 months of continuous monitoring. This is for instance the type of monitoring planned in the "Super-Earth Explorer" project (Schneider et al. 2006). For TPF-C, the collecting area is 20 times larger and correspondingly the exposure time to detect a $0.3 R_{\text {Jup }}$ companion drops to $30 \mathrm{~h} / 20=1.5 \mathrm{~h}$. Note that a continuous monitoring is also required for the detection of surface inhomogeneities of planets (Ford et al. 2001).

\subsection{Geometric probability of shadows and transits and observation strategy}

The geometric probability of mutual transits is $p=R_{\mathrm{pl}} / a_{\mathrm{sat}}$. For a Saturn/Titan-like configuration it is approximately $10 \%$. The probability that a planet and its companion make mutual eclipses (with respect to the parent star) depends on the inclination of the orbital plane of the planet-companion system with respect to the orbit of the system around the parent star. If both objects have been formed by accretion in a protoplanetary disk, it it is likely that the two orbital planes are close to each other. Thus mutual shadows should occur at any position of the planet-companion orbit around the star. Consequently, the geometric probability of mutual shadows should be nearly $100 \%$.

For mutual shadows, which have the maximum geometric probability of occurrence, the total fraction of time for which the event occurs is:

$$
\frac{\Delta T_{1}+\Delta T_{3}}{P_{\mathrm{comp}}} \approx \frac{3}{\pi} \frac{R_{\mathrm{pl}}}{a_{\mathrm{comp}}}
$$

It is of the order of $10 \%$ for a Saturn-Titan system. It is thus necessary to have a duty cycle of at least $90 \%$ in order not to miss this event. The duration of this high duty cycle must be at least $P_{\text {comp }}$, i.e. 15 days for a Saturn-Titan system. In conclusion, 
the detection of companions by mutual shadows (the most probable event) requires continuous imaging of the planet for at least about two weeks. Such continuous imaging is possible only from space.

\section{References}

Ardeberg, A. 2005, in The Science Case for the European Extremely Large Telescope: the Next Step in Mankind's Quest for the Universe, ed. I. Hook, 16

Arnold, L., \& Schneider, J. 2004, A\&A, 420, 1153

Barnes, J. W., \& O'Brien, D. P. 2002, ApJ, 575, 1087

Barnes, J. W., \& Fortney, J. J. 2004, ApJ, 616, 1193

Bennett, D. P., \& Rhie, S. H. 2002, ApJ, 574, 985

Brown, T. M., Charbonneau, D., Gililand, R. L., Noyes, R. W., \& Burrows, A. 2001, ApJ, 552, 699

Canup, R., \& Ward, W. 2006, Nature, 441, 834

Fairbairn, M. B. 2002, JRASC, 96, 18

Ford, E., Seager, S., \& Turner, E. 2001, Nature, 412, 885

Gaidos, E., Moskovitz, N., \& Williams, D. M. 2006, Terrestrial Exoplanet Light Curves, in Direct Imaging of Exoplanets: Science \& Techniques, ed. C. Aime, \& F. Valiki (Cambridge University Press), Proc. IAU Coll., 200, 153
Guillot, T. 2005, Ann. Rev. Earth \& Plan. Sci. 33, 493

Han, C., \& Han, W. 2002, ApJ, 580, 490

Ida, S., Canup, R., \& Stewart, G. 1997, Nature, 389, 353

Kern, S., \& Elliot, J. 2006, Icarus, 183, 179

Kley, W. 2003, Evolution of Planets in Disks. In Towards Other Earths: DARWIN/TPF and the Search for Extrasolar Terrestrial Planets, ESA SP539, 163

Lester, T. P., McCall, M. L., \& Tatum, J. B. 1979, JRASC, 73, 234

Pravec, P., Scheirich, P., Kusnirak, P., et al. 2006, Icarus, 181, 63

Sartoretti, P., \& Schneider, J. 1999, A\&AS, 134, 553

Schneider, J. 2003, in Towards Other Earths: DARWIN/TPF and the Search for Extrasolar Terrestrial Planets, ESA SP-539

Schneider, J., Riaud, P., Tinetti, G., \& the SEE-COAST Team 2006. The SuperEarth Explorer, in Proceedings of the Société Française d'Astronomie et d'Astrophysique, ed. D. Barret, et al. in press

Spencer, J. 1987, The Surfaces of Europa, Ganymede and Callisto: an Investigation Using Voyager IRIS Thermal Infrared Spectra, Ph.D. Thesis, Univ. of Arizona

Stassun, K., Mathieu, R., \& Valenti, J. 2006, Nature, 440, 311

Teolis, B., Loeffler, M., Raut, U., et al. 2006, ApJ, 664, L141

Valtonen, M., \& Karttunen, H. 2006, in The Three-Body Problem (Cambridge: Cambridge University Press)

Williams, D., \& Knacke, R. 2004, Astrobiology, 4, 400 